\title{
ANALYTICA
}

CHIMICA

ACTA

ELSEVIER

Analytica Chimica Acta 426 (2001) 111-117

www.elsevier.com/locate/aca

\section{Capillary liquid chromatographic determination of neutral phenolic compounds in apple juices}

\author{
Domingo Blanco Gomis*, Natalia Fraga Palomino, Juan José Mangas Alonso \\ Departamento de Química Física y Analítica, Universidad de Oviedo, 33006 Oviedo, Spain
}

Received 23 May 2000; received in revised form 7 August 2000; accepted 31 August 2000

\begin{abstract}
Capillary liquid chromatography (CLC) is evaluated as an alternative to conventional HPLC to analyse complex phenolics. Several neutral phenolic compounds were separated on a packed-reversed phase fused-silica capillary column, and determined with UV detection. A conventional liquid chromatographic equipment was adapted for such purposes. Application of the proposed method to the quantification of neutral phenols in apple juices is reported. Phenolics are extracted and fractionated into neutral and acidic compounds by means of a $\mathrm{C}_{18}$ solid-phase cartridge. Typical recoveries ranging from 90 to $105 \%$ are obtained and reproducibility between extractions is $<7 \%$ in all cases. Mass detection limits are at the sub-nanogram level. (C) 2001 Elsevier Science B.V. All rights reserved.
\end{abstract}

Keywords: Polyphenols; Apple juices; Capillary liquid chromatography

\section{Introduction}

Phenolic compounds in apples are of undoubted importance because of their contribution to the colour, taste and flavour characteristics of apples and its derivative products $[1,2]$, their use as indicators of the physiological stages during fruit development and their participation in technological processes leading to low quality products and in possible alterations (browning, formation of hazes and sediments, etc.) [3]. Recently, polyphenols have been the object of increasing interest because of their biological properties, namely anti-inflammatory, anti-histaminic and anti-tumor activities, and as free radical scavengers and protection against cardiovascular diseases [4-7].

\footnotetext{
${ }^{*}$ Corresponding author. Tel.: +34-985-103490; fax: +34-985-103125.

E-mail address: dbg@sauron.quimica.uniovi.es (D.B. Gomis).
}

Due to the structural complexity of polyphenols and the great variability that exists, the use of modern analytical techniques is required for their analysis. In this sense, HPLC techniques have proved to be the most appropriate ones, allowing polyphenol analysis with sufficient precision, sensitivity and within a reasonable time.

Microcolumn liquid chromatography, also known as capillary liquid chromatography (CLC), started to develop at the same time as wide-bore (conventional) HPLC. However, slow progress in the necessary instrumentation has kept CLC as a research topic more than a practical tool in the laboratory. Only recently, due to the availability of commercial microcolumns and the progress in the instrumentation, it is possible to study its ability as an alternative to conventional HPLC.

Theoretically, the use of capillary columns, with internal diameters lower than $500 \mu \mathrm{m}$, would present several analytical advantages (increase in mass sensi- 
tivity, high resolution and easy coupling to detectors and secondary chromatographic systems).

Besides, the cost per analysis is drastically reduced when working with flow rates between 1 and $5 \mu \mathrm{l} / \mathrm{min}$, requiring only nanolitres of sample [8-12].

Moreover, low molecular mass polyphenols are also being used for the typification of foods and some of them have been used to determine the authenticity of fruit products, detecting possible adulterations [13-15]. To be able to do this, it is necessary to have data on the quantity and type of phenolic compounds. The main limitation is that of the problem of polyphenolic identification due to the lack of commercial standards for some of these compounds, being necessary to carry out their isolation before the analysis, usually employing tedious and time-consuming techniques. In the last few years, mass spectrometry (MS) coupled to HPLC has become in one of the more powerful identification techniques being the low diameter chromatographic columns the more appropriate ones for this purpose, as they allow a direct coupling. Herein we find another utility of this capillary liquid technique.

This paper reports the CLC determination of several neutral phenolic compounds. They were separated and quantitated in apple juices using UV detection. The evaluation of the method (detection limits, linearity, reproducibility and recoveries) confirm that CLC can be employed as an alternative to conventional HPLC chromatography to analyse these compounds in apple samples.

\section{Experimental}

\subsection{Apparatus}

A conventional HPLC apparatus was suited to practise the capillary HPLC technique. The micro-LC equipment consisted of two Kontron 422 pumps (Kontron Instruments, Milan, Italy) connected to a T-flow-split system which enabled the mobile phase to be distributed between the by-pass and the microcolumn, connected to a zero-dead volume Valco Injector equipped with a $60 \mathrm{nl}$ internal loop (Valco, Houston, TX, USA). Eight determinations of the standards retention times within twenty days offered an average R.S.D. of $2 \%$. This value indicate good flow repeatability with this chromatographic design.
The microcolumn used was a Hypersil ODS2 $(250 \mathrm{~mm} \times 0.30 \mathrm{~mm}$ i.d.; $5 \mu \mathrm{m}$ particle size $)$, from LC Packings, Amsterdam, The Netherlands. It was directly connected to the injection valve in order to minimise extra-column dead volumes.

Analysis of the samples were carried out at room temperature. Detection was performed at $280 \mathrm{~nm}$ with a Kontron model 430 UV-VIS variable wavelength detector, provided with a $90 \mathrm{nl}, 20 \mathrm{~mm}$ flow cell, coupled to a Kontron Instruments System 450 Data Treatment Station.

\subsection{Chemicals}

Standards of (+)-catechin, (-)-epicatechin, phloridzin and chlorogenic acid were obtained from Sigma Chemical (St. Louis, MO, USA), glycosides of quercetin (isoquercetin, hyperin, quercitrin, avicularin and rutin) were from Extrasyntèse (Genay, France), procyanidins and phloretin xyloglucoside were kindly furnished by Dr. A. Lea (Reading, UK). Polyphenol standard solutions were prepared in methanol and stored at $-20^{\circ} \mathrm{C}$. Solvents employed were from Romil (Loughborough, UK), HPLC quality and degassed with helium prior to use. High purity water was obtained through a Millipore Milli-Q system (Milford, MA, USA).

\subsection{Samples}

Simple-variety apple juices were obtained by means of a domestic juice-maker and centrifuged at $12,000 \mathrm{~g}$ for $5 \mathrm{~min}$. Other apple juices were obtained by pressing from a mild-sharp cider apple juice mixture and stabilised by microfiltration through mineral membranes of $0.14 \mu \mathrm{m}$ pore size. All juices were frozen at $-20^{\circ} \mathrm{C}$ until their analytical determination.

\subsection{Solid-phase extraction of phenolics and sample preparation}

Solid-phase extraction (SPE) was carried out using a Visiprep Vacuum manifold (Supelco, Bellefonte, PA), a Vacuumbrand $\mathrm{GmbH}$ (Wertheim, Germany) pump and two different $\mathrm{C}_{18}$ solid-phase supports were compared: Mega Bond Elut $\mathrm{C}_{18}(1000 \mathrm{mg}$; Varian, Harbor City, CA, USA) and Extra-Sep $\mathrm{C}_{18}$ (1000 mg; 
Lida, Kenosha, WI, USA), which was the one finally chosen.

Both supports were preconditioned, according to the method optimised by Suárez et al. [16], sequentially passing $10 \mathrm{ml}$ of methanol and $5 \mathrm{ml}$ of water adjusted to $\mathrm{pH}$. Then, a suitable volume of the sample $(3-4 \mathrm{ml}$, in order to load a maximum of $8 \mathrm{mg}$ of total polyphenols per gram of solid-phase) containing $10 \mathrm{mg} / \mathrm{l}$ of ascorbic acid to avoid the oxidation of polyphenols, was adjusted to $\mathrm{pH} 7$ with $\mathrm{NaOH}$, loaded into the previously preconditioned column and washed with $13 \mathrm{ml}$ of $\mathrm{pH} 7$ water, to eliminate sugars, phenolic acids and organic acids. The adsorbed fraction was eluted with $16 \mathrm{ml}$ of methanol. The organic solvent was removed using a rotatory evaporator at $30^{\circ} \mathrm{C}$ and the residue diluted in $0.5 \mathrm{ml}$ of methanol, filtered through a $0.22 \mu \mathrm{m}$ filter and finally, $60 \mathrm{nl}$ were injected into the HPLC system.

\subsection{Chromatographic conditions}

The binary gradient used at a flow-rate of $3 \mu \mathrm{l} / \mathrm{min}$ was as follows: $0 \mathrm{~min}, 2 \% \mathrm{~B} ; 0-42 \mathrm{~min}, 35 \% \mathrm{~B}$; 42-60 min, 35\% B; 60-75 min, 46\% B; 75-85 min, $52 \% \mathrm{~B}$, where solvent $\mathrm{A}$ was water adjusted to $\mathrm{pH} 2.5$ with phosphoric acid and solvent $\mathrm{B}$ was methanol.

Identification of the compounds was performed by means of their retention time and UV spectra, as the sofware of the UV-VIS variable wavelength detector allows to make fast sweeps of the spectra, and by spiking with the standards when available. Spectra were taken at the leading edge, the apex and the tailing edge to monitor for peak purity. Knowledge of the spectra of unknown chromatographic peaks can also give a preliminary idea about the chemical structure of the corresponding compound. In this way, four chromatographic peaks could be assign to different phenolic families (cinnamic acid derivatives, procyanidins and flavonol glycosides) with characteristic UV spectra.

Quantitation was carried out at $280 \mathrm{~nm}$ by the external standard method. For the compounds lacking standards or those for which the amount at our disposal was too small, quantitation was carried out with similar compounds. Thus, procyanidins were quantified as (-)-epicatechin, phloretin xyloglucoside as phloridzin and the unknown flavonol glycoside as rutin.

\subsection{Recovery studies}

Recovery experiments were performed in order to study the accuracy of the method described. Known amounts of pure standards ((+)-catechin, (-)-epicatechin, phloridzin, avicularin and quercitrin) were added to a variety of samples and the resulting spiked samples were subjected to the entire analytical sequence, including the extraction step. Each compound was added at three different concentrations and recoveries were calculated on the basis of the difference between the total amount determined in the spiked samples and the amount observed in the non-spiked samples. All analyses were carried out in triplicate.

\section{Results and discussion}

\subsection{Optimisation of chromatographic conditions}

In order to separate the neutral phenolic compounds in apple juices (flavonoids and hydroxycinnamic acids derivatives with sugars), we chose a Hypersil ODS2 $(250 \mathrm{~mm} \times 0.30 \mathrm{~mm}$ i.d.; $5 \mu \mathrm{m}$ particle size $)$ microcolumn, since it was the only reversed-phase available packing. There were neither at that time capillary columns with particle size $<5 \mu \mathrm{m}$.

The main chromatographic conditions to be optimised were the flow rate, $\mathrm{pH}$ and the percentage of the organic modifier of the mobile phase.

It is well-known that these types of microcolumns show similar efficiencies working with flow rates between 1 and $10 \mu \mathrm{l} / \mathrm{min}$. Therefore, the highest value in this interval allowed by the pressure reached should be chosen in order to shorten the analysis time. The parameter to be optimised is the flow rate imposed on the pumps as a function of the split system employed. The best option was found to be $3 \mu \mathrm{l} / \mathrm{min}$, obtained with the pumps operating at a flow rate of $0.1 \mathrm{ml} / \mathrm{min}$ and a Spherisorb ODS $(25 \mathrm{~cm} \times 0.4 \mathrm{~cm}$ i.d.; $3 \mu \mathrm{m}$ particle size) column working as a pressure restrictor.

With regards to the $\mathrm{pH}$, this must be adjusted to a sufficiently low value so as to ensure the protonation of the compounds during the whole analysis, employing an acid (in this case, phosphoric acid) better than a buffer solution, as previous studies [17] showed that an increase in ionic strength brought about longer 

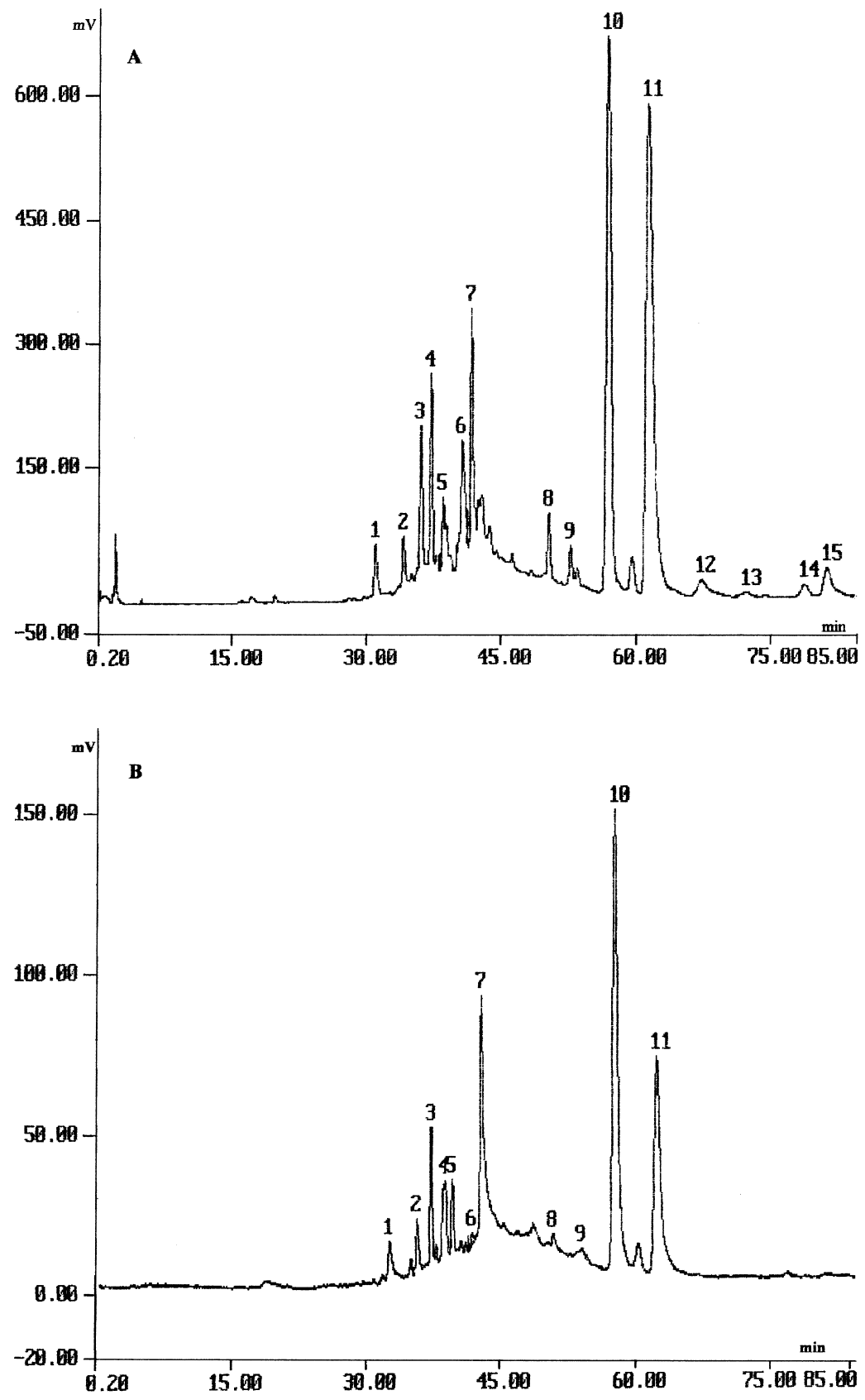

Fig. 1. Separation of neutral phenolic compounds in a simple-variety apple juice (A) and in a cider apple mixture juice stabilised by microfiltration through mineral membranes (B). Peaks are indicated as follows: (1) procyanidin B1; (2) (+)-catechin; (3) cinnamic ester, (4) procyanidin B2; (5) chlorogenic acid; (6) trimer $\mathrm{C} 1+$ tetramer; (7) (-)-epicatechin; (8) unknown procyanidin; (9) unknown procyanidin; (10) phloretin xyloglucoside; (11) phloridzin; (12) rutin + hyperin + isoquercitrin; (13) avicularin; (14) unknown flavonol; (15) quercitrin. Compounds (3), (8), (9) and (14) were recognised due to their UV spectrum. Chromatographic conditions as given in Section 2.5. 
Table 1

Physical characteristics of the packing materials employed and its effect on the percentage of chlorogenic acid remaining with the neutral fraction

\begin{tabular}{llllcc}
\hline Solid-phase supports & Particle size $(\mu \mathrm{m})$ & Porosity $(\AA)$ & Endcapped & Carbon loading $(\%)$ & Chlorogenic acid $(\%)$ \\
\hline Mega Bond Elut $\mathrm{C}_{18}$ & 40 & 60 & Yes & 18 & 5.3 \\
Extra-Sep C 18 & 50 & 60 & Yes & 6 & 4.2 \\
\hline
\end{tabular}

retention times and worse resolution. Optimisation of this parameter was carried out in the range 2.5-3.4 and a $\mathrm{pH}$ of 2.5 was chosen for the analysis. It must be pointed out that chlorogenic acid was included in the study in spite of its acidic nature because part of it is expected to remain with the neutral fraction.

The optimised gradient elution is shown in Section 2. Good resolution is found for all the compounds, except for the procyanidins $\mathrm{C} 1$ and a tetramer and the flavonols rutin, hyperin and isoquercitrin (Fig. 1). In relation to the long analysis time and the lack of identifiable peaks for the first $30 \mathrm{~min}$, when the strength of the solvent was altered initially or the percentage of methanol was faster increased to elute interested compounds early and decrease the analysis time, worse separation of polyphenol compounds was obtained.

Similar resolutions and analysis times have also been reported for similar stationary phases [17-19]. Other authors describe faster elution gradients, with analysis times among 55 and $70 \mathrm{~min}$, but they do not quantify compounds such as trimer $\mathrm{C} 1$ and tetramer [20], tetramer and flavonols [21] or all the procyanidins [15]. Nowadays, fast chromatographic methods for apple phenolics have been published [22,23], employing a Nucleosil $120 \mathrm{C}_{18}$ column, which has been yet reported as a better RP-packing for these type of compounds. Nevertheless, compounds as trimer $\mathrm{C} 1$ and tetramer are neither included in these works.

\subsection{Extraction conditions}

Two $\mathrm{C}_{18}$ solid-phase supports, Extra-Sep $\mathrm{C}_{18}$ (1000 mg; Lida, Kenosha, WI, USA) and Mega Bond Elut $\mathrm{C}_{18}(1000 \mathrm{mg}$; Varian, Harbor City, CA) were tested. As shown in Table 1, the Extra-Sep $\mathrm{C}_{18}$ column offered a high fractionation capacity, the percentage of the acidic compounds remaining in the neutral fraction being lower. These differences might be explained by the different physical characteristics of the packing materials. Thus, the higher carbon loading of the Mega Bond Elut $\mathrm{C}_{18}$ cartridges might account for the greater retention of the phenolic acids, as a linear relationship between carbon loading and capacity factor has been described [24]. The Extra-Sep $\mathrm{C}_{18}$ was hence the one chosen to perform the analysis under the conditions described above.

\subsection{Analytical performance}

Calibration plots were generated by repeated injections of $60 \mathrm{nl}$ of standard solutions of phenolics at five different concentrations. The calibration graphs for all the studied compounds showed good correlation between the peak areas and phenolic concentrations, with regression coefficients $>0.999$ in all cases, from the determination limit up to the investigated concentration limit, as it is shown in Table 2.

Table 2

Analytical characteristics of the calibration graphs

\begin{tabular}{lllclr}
\hline Analyte & Linear range $(\mathrm{mg} / \mathrm{l})$ & Slope & Intercept & $r^{\mathrm{a}}$ & Detection limit $(\mathrm{pg})$ \\
\hline (+)-Catechin & $3.0-350$ & 0.15 & 0.03 & 0.9994 & 97 \\
(-)-Epicatechin & $0.6-1000$ & 0.28 & -0.01 & 0.9999 & 20 \\
Phloridzin & $0.3-1800$ & 0.39 & 0.01 & 0.9999 & 9 \\
Rutin & $0.3-50$ & 1.53 & 0.02 & 0.9999 & 10 \\
Avicularin & $0.5-20$ & 0.60 & 0.01 & 0.9991 & 15 \\
Quercitrin & $0.9-50$ & 1.57 & -0.04 & 0.9990 & 32 \\
\hline
\end{tabular}

\footnotetext{
${ }^{\mathrm{a}}$ Correlation coefficients of the regression equation $y=a+b x$, where $x$ is the phenolic compound concentration (ppm) and $y$ the peak
} area. 
Table 3

Recoveries of phenolic compounds from an apple juice

\begin{tabular}{lc}
\hline Analyte & Mean recovery $(\%) \pm$ R.S.D. \\
\hline (+)-Catechin & $100 \pm 4$ \\
$95 \pm 4$ \\
$99 \pm 5$ \\
(-)-Epicatechin & $102 \pm 7$ \\
& $98 \pm 2$ \\
& $103 \pm 3$ \\
Phloridzin & $101 \pm 6$ \\
& $100 \pm 2$ \\
Avicularin & $101 \pm 4$ \\
& $104 \pm 4$ \\
Quercitrin & $103 \pm 6$ \\
& $94 \pm 4$ \\
& $96 \pm 2$ \\
& $105 \pm 1$ \\
\hline
\end{tabular}

${ }^{\mathrm{a}}$ Mean values and relative standard deviation for three replicates.

The mass limits of detection, based on a signal-tonoise ratio of 3 , were in the level of the sub-nanogram for all the compounds. The values obtained are summarised in Table 2 and ranged from $9 \mathrm{pg}$ for phloridzin to $97 \mathrm{pg}$ for $(+)$-catechin.

The average recoveries, obtained as indicated in Section 2.6 and given in Table 3, ranged between 90 and $105 \%$. These values testify to the accuracy of the proposed method. The precision of the method was investigated with real samples. The R.S.D.'s for the sample were determined using three different apple juice samples and analysing each sample five times, and were always $<7 \%$.

\subsection{Application}

As an example of the application to real samples, Fig. 1 shows the chromatograms corresponding to the neutral phenolic compounds of a simple-variety apple juice obtained by means of a domestic juice-maker (A) and to a juice obtained by pressing from a mild-sharp cider apple mixture and stabilised by microfiltration through mineral membranes of $0.14 \mu \mathrm{m}$ pore size (B), both of them obtained by using the established conditions.

Contents of the quantified compounds are given in Table 4. All samples were analysed in triplicate and R.S.D.'s were always less than 3.5\%. It can be noticed that, although the profiles of the phenolic compounds are similar, the microfiltered juice has a lower polyphenol content. This fact could be related to the greater degree of oxidation and the adsorption ability of the cake layer developed on the membrane microfiltration surface [25]. Likewise, the low content of flavonols detected in the stabilised apple juices could be linked to the pressing system employed, as the use of an hydraulic rack and frame press does not allow them to be adequately extracted.

Table 4

Content $(\mathrm{mg} / \mathrm{l})$ of neutral polyphenols in two different simple-variety apple juice (San Pedro and San Juan) and a microfiltered stabilised juice

\begin{tabular}{lclc}
\hline Compound & Apple juice 1 (San Pedro) & Stabilised apple juice & Apple juice 2 (San Juan) \\
\hline Procyanidin B1 & 10.8 & 2.3 & 15.1 \\
(+)-Catechin & 17.7 & 3.8 & 28.6 \\
Procyanidin B2 & 33.4 & 8.8 & 63.5 \\
Trimer C1 + tetramer & 34.6 & 1.9 & 42.1 \\
(-)-Epicatechin & 34.3 & 15.6 & 83.6 \\
Unknown procyanidin & 12.4 & 1.9 & 9.9 \\
Unknown procyanidin & 9.6 & 1.3 & 11.4 \\
Phloretin xyloglucoside & 107.2 & 30.9 & 151.6 \\
Phloridzin & 147.9 & 27.4 & 159.2 \\
Rutin + hyperin + isoquercitrin & 1.7 & n.d. & 1.9 \\
Avicularin & 0.9 & n.d. & 0.9 \\
Unknown flavonol & 1.6 & n.d. & 1.8 \\
Quercitrin & 2.3 & n.d. & 3.4 \\
\hline
\end{tabular}

${ }^{a}$ n.d.: Not detected. 
In agreement with some authors [16,18,22], higher concentrations of dyhidrocalchones than flavanols are found. Nevertheless, another works $[14,26]$ indicate just the opposite. It suggests that the levels of phenolic compounds in apple and apple juices depend strongly on the fruit variety.

\section{Conclusions}

Experimental results suggest this capillary liquid chromatographic method is appropriate for analysing neutral polyphenols in apple juices, showing a resolution similar to that offered by conventional columns and very low mass detection limits. Moreover, the use of flows in the range of $1-5 \mu \mathrm{l} / \mathrm{min}$ would allow a direct coupling to mass spectrometry and, on the other hand, if a great number of analyses is required, costs will be drastically reduced.

\section{Acknowledgements}

The authors are indebted to the Comisión Interministerial de Ciencia y Tecnología (CICYT, Project ALI 92-1027-CO3) for financial support and the Fundación para la Investigación Científica y el Desarrollo Tecnológico (FICYT) of the Principado de Asturias for a grant to Dña. Natalia Fraga Palomino.

\section{References}

[1] A.G.H. Lea, G. Arnold, J. Sci. Food Agric. 29 (1978) 478.

[2] A. Bilyk, K.B. Hicks, D.D. Bills, G.M. Sapers, J. Liq. Chromatogr. 11 (1988) 2829.

[3] J.J. Macheix, A. Fleuriet, J. Billot, Fruit Phenolics, CRC Press, Boca Raton, FL, 1990 (Chapter 4).
[4] M. Bourzeix, Polyphenolic Phenomena, INRA Editions, París, 1993 , p. 287.

[5] D. Treutter, W. Fencht, J. Hort. Sci. 65 (1990) 511.

[6] J.A. Vinson, B. Hontz, J. Agric. Food Chem. 43 (1995) 401.

[7] J.A. Vinson, Y.A. Dabbagh, M.M. Serry, J. Jang, J. Agric. Food Chem. 43 (1995) 2800.

[8] M. Krejcí, Chromatographic Science Series, Vol. 599, Marcel Dekker, New York, 1992.

[9] M. Novotny, in: Proceedings of the 13th International Sysmposium on Capillary Chromatography, Riva del Garda, Italy, 13-16 May 1991.

[10] C.E. Reese, R.P.W. Scott, J. Chromatogr. Sci. 18 (1980) 479

[11] M. Novotny, Anal. Chem. 60 (1988) 500A.

[12] D. Ishii, T. Tsuda, T. Nakanishi, J. Chromatogr. 185 (1979) 73.

[13] B. Fernández de Simón, J. Pérez-Ilzarbe, T. Hernández, C. Gómez-Cordovés, I. Estrella, J. Agric. Food Chem. 40 (1992) 1531.

[14] G.A. Spanos, R. Wrolstad, J. Agric. Food Chem. 40 (1992) 1478.

[15] P.B. Andrade, A.R.F. Carvalho, R.M. Seabra, M.A. Ferreira, J. Agric. Food Chem. 46 (1998) 968.

[16] B. Suárez, A. Picinelli, J.J. Mangas, J. Chromatogr. A 727 (1996) 203.

[17] B. Suárez, J. Santamaría, J.J. Mangas, D. Blanco, J. Agric. Food Chem. 42 (1994) 2732.

[18] E. Delage, G. Bohuon, A. Baron, J.F. Drilleau, J. Agric. Food Chem. 551 (1991) 125.

[19] J. Pérez-Ilzarbe, T. Hernández, Y. Estrella, Z. Lebensm Unters Forsch 192 (1991) 551.

[20] M.L. Bengoechea, A.I. Sancho, B. Bartolomé, Y. Estrella, C. Gómez Cordovés, M.T. Hernández, J. Agric. Food Chem. 45 (1997) 4071.

[21] J.J. Mangas, R. Rodríguez, B. Suárez, A. Picinelli, E. Dapena, J. Agric. Food Chem. 47 (1997) 4046.

[22] A. Escarpa, M.C. González, J. Chromatogr. A 823 (1998) 331.

[23] A. Escarpa, M.C. González, J. Chromatogr. A 830 (1999) 301.

[24] C. Horváth, in: C.F. Simpson De (Ed.), Techniques in L.C., Wiley, New York, 1982 (Chapter 10).

[25] A. Picinelli, B. Suárez, J.J. Mangas, Z. Lebensm Unters Forsch A 204 (1997) 48.

[26] G.A. Spanos, R.E. Wrolstad, D.A. Heatherbell, J. Agric. Food Chem. 38 (1990) 1572. 